\title{
Cyclotron resonance of a two-dimensional Wigner solid
}

\author{
Yu. P. Monarkha \\ B. Verkin Institute for Low Temperature Physics and Engineering of the National Academy of Sciences of Ukraine \\ 47 Lenin Ave., 61103 Kharkov, Ukraine \\ E-mail: monarkha@ilt.kharkov.ua
}

Received Jabuary 15, 2001, revised February 12, 2001

\begin{abstract}
Cyclotron resonance absorption from a two-dimensional electron solid formed on a free surface of liquid helium is analyzed by means of the memory function formalism. The presence of the low-frequency phonon mode of the Wigner solid in a strong magnetic field is show to broaden significantly the electron dynamical structure factor (DSF) as a function of frequency, strengthening the contribution from multi-phonon emission terms of the high-frequency mode. Thus in most cases the linewidth is formed by nonlinear terms of the DSF expansion in the cyclotron motion factor $\exp \left(-i \omega_{c} t\right)$ rather than by the linear terms analyzed previously. The inclusion of all these multi-phonon terms changes the sign of the many-electron effect and agrees well with available experimental data, eliminating the discrepancy between theory and experiment.
\end{abstract}

PACS: $73.20 . \mathrm{Dx}, \mathbf{7 3 . 2 5 . + i , 7 6 . 4 0 . + b}$

\section{Introduction}

Coulomb forces can strongly affect the properties of a two-dimensional (2D) electron system. An extreme example of this kind is the Wigner solid transition. The Wigner solid (WS) of a 2D nondegenerate electron gas was firstly observed in a sheet of electrons formed on a free surface of liquid helium $[1,2]$. The long-range order of a finite $2 \mathrm{D}$ electron system was detected by a resonance coupling of the electron lattice with capillary wave quanta (ripplons).

Significant research has been performed on using the cyclotron resonance (CR) as a probe of the many-electron effects and WS phase. A strong narrowing of the CR linewidth was observed in semiconductor degenerate 2D electron systems [3,4] for small filling factors. It should be noted that in degenerate systems, the influence of Coulombic effects increases with lowering electron density $n_{s}$. A detailed experimental study of CR absorption from surface electrons (SEs) on liquid helium was reported in $[5,6]$, though by that time the many-electron theory were not enough developed to explain these data. For SEs on liquid helium the Fermi energy is very small $\varepsilon_{F}<T$, and increasing the electron density introduces many-electron effects. Some data of Refs. 5, 6 had shown a decrease of the
CR linewidth with increase of the holding electric field $E_{\perp}$, which was maintained under the saturation condition: $E_{\perp}=2 \pi e n_{s}$. However, at low temperatures, the holding electric field acts also as an electron-ripplon coupling parameter affecting directly the CR linewidth.

A CR theory of a $2 \mathrm{D}$ electron crystal was introduced in Ref. 7. According to it, the CR linewidth is due to decay of the long-wavelength $(k \rightarrow 0)$ high-frequency phonon $\Omega_{+, k} \simeq \omega_{c}$ into a short-wavelength phonon of the same branch accompanied by the creation and annihilation of a great number of low-frequency phonons $\Omega_{-, k} \simeq \omega_{l, k} \omega_{t, k} / \omega_{c}<<$ $\ll T / \hbar$ [here $\omega_{p, k}$ is the spectrum of longitudinal $(p=l)$ and transverse $(p=t)$ phonons in the absence of the magnetic field, and $\omega_{c}$ is the cyclotron frequency]. The WS theory [7] was confined to analysis of the linear terms of the conductivity expansion in the cyclotron motion factor $\exp \left(-i \omega_{c} t\right)$, which is similar to considering only one-phonon terms in the dynamical structure factor (DSF) of a solid. This approximation resulted in a remarkable conclusion: the CR linewidth $\gamma_{C R}$ decreases with electron density as $\gamma_{C R} \propto n_{s}^{-3 / 4}$ [7]. This result was used to explain the narrowing of the $\mathrm{CR}$ absorption line reported earlier $[5,6]$. A similar result was found employing the path-integral method [8]. 
A direct check of the theory [7] performed for SEs at low temperatures $(T=0.062 \mathrm{~K})$ and relatively weak magnetic fields $\left(B \simeq 0.071 \mathrm{~T}, \hbar \omega_{c} / T \simeq\right.$ $\simeq 1.55)$ has strikingly shown an opposite density dependence: the linewidth increases with electron density approximately as $\gamma_{C R} \propto n_{s}$ [9]. In this experiment, the electron density was varied independently of the holding electric field. For a liquid state of the electron system the explanation of this conflict between experiment and theory was found in Refs. 10, 11: the ultrafast motion of electron orbit centers in a fluctuational many-electron field $\mathbf{E}_{f}$ stimulates electron scattering between different Landau levels, causing an increase of the CR linewidth. This picture describes nicely the CR absorption from SEs on liquid helium interacting with helium vapor atoms. Still, it is of interest to find out how this concept changes for the WS state, and what revision of the WS theory should be introduced.

In this paper, to describe the CR of the WS we employ the memory function formalism [12,13], which allows one to find a relation between the CR linewidth and the electron solid DSF $S(q, \omega)$. The analysis of the Wigner solid DSF in the presence of a high magnetic field performed here shows that for strong Coulomb forces the nonlinear terms of the expansion in the cyclotron motion factor $\exp \left(-i \omega_{c} t\right)$ cannot be disregarded. In other words, multi-highfrequency-phonon terms crucially affect the CR linewidth if $\Gamma_{C} \equiv \sqrt{2} e E_{f} l>\hbar \omega_{c}$ (here $l=\sqrt{\hbar c / e B}$ is the magnetic length). The strong broadening $\Gamma_{C}$ of the DSF $S(q, \omega)$ caused by the low-frequency mode $\Omega_{-, k}$, makes it possible to emit many high-frequency phonons $\sum \Omega_{+, k} \simeq N \omega_{c}$, a process which is equivalent to the electron scattering on higher Landau levels introduced for the electron liquid CR theory [10]. When all of the nonlinear terms $\exp \left(-i \omega_{c} t N\right)$ are taken into account, the Wigner solid DSF is found to be similar to the DSF of a highly correlated electron liquid reported in Ref. 11. The result of evaluation of the CR linewidth induced by the electron-ripplon scattering agrees well with experimental data of Refs. 5, 6 and 9 , eliminating the conflict between theory and experiment.

\section{Basic relations}

The memory function formalism [12,13] establishes a general relation between the electron conductivity $\sigma$ and the memory function or the relaxation kernel $M(\omega)$. The effective collision frequency can be introduced as $v_{\text {eff }}=\operatorname{Im}[M(\omega)]$. Searching for the CR linewidth, we disregard $\operatorname{Re}[M(\omega)]$ or consider that it is included somehow in the definition of the resonance frequency. In this case, we have

$$
\sigma_{x x} \pm i \sigma_{x y}=\frac{i e^{2} n_{s}}{m\left[\omega \mp \omega_{c}+i v_{\mathrm{eff}}(\omega)\right]}
$$

In the conventional procedure [12], $M(\omega)$ is expressed in terms of the force-force Green's function. For $\operatorname{Im}[M(\omega)]$ the final equation can be simplified significantly by employing general properties of the Green's function,

$$
\begin{gathered}
\nu_{\text {eff }}(\omega)= \\
=-\frac{[1-\exp (-\hbar \omega / T)] m}{2 e^{2} n_{s} \hbar^{3} \omega S_{A}} \int_{-\infty}^{\infty}\left\langle F_{x}(t) F_{x}\right\rangle \exp (i \omega t) d t,
\end{gathered}
$$

where $S_{A}$ is the surface area, $F_{\beta}=i\left[J_{\beta}, H_{\text {int }}\right]$ is the force acting on electrons (with a factor $e / m$ ), determined by the interaction Hamiltonian $H_{\text {int }}$ and current density $\mathbf{J}=-\sum_{s} e \mathbf{v}_{s}$.

Ripplons represent a sort of $2 \mathrm{D}$ phonons with the capillary spectrum $\omega_{q}=\sqrt{\alpha / \rho} q^{3 / 2}$ (here $\alpha$ is the surface tension and $\rho$ is the density of liquid helium). On introducing a $2 \mathrm{D}$ electron density operator $n_{\mathbf{q}}=\sum_{s} \exp \left(-i \mathbf{q r}_{s}\right)$ and the interaction Hamiltonian

$$
\begin{gathered}
H_{\text {int }}=\frac{1}{\sqrt{S_{A}}} \sum_{\mathbf{q}} U_{q} n_{-\mathbf{q}}\left(b_{\mathbf{q}}+b_{-\mathbf{q}}^{+}\right), \\
U_{q}=V_{q} \sqrt{\hbar q / 2 \rho \omega_{q}},
\end{gathered}
$$

the force operator can be found as [14]

$$
F_{\beta}=-\frac{i \hbar e}{m \sqrt{S_{A}}} \sum_{\mathbf{q}} q_{\beta} U_{q^{n}-\mathbf{q}}\left(b_{\mathbf{q}}+b_{-\mathbf{q}}^{+}\right) .
$$

Here $b_{\mathbf{q}}$ is the ripplon annihilation operator.

The particular form of the electron-ripplon coupling $V_{q}$ is taken from Ref. 15:

$$
V_{q}=e E_{\perp}+\frac{\Lambda q^{2}}{2} w\left(\frac{q^{2}}{4 \gamma_{\perp}^{2}}\right)
$$

where 


$$
\omega(x)=-\frac{1}{1-x}+\frac{1}{(1-x)^{3 / 2}} \ln \left(\frac{1+\sqrt{1-x}}{\sqrt{x}}\right),
$$

$\gamma_{\perp}^{-1}$ is the localization length of the electron wave function in the perpendicular direction, $\Lambda=$ $=e^{2}(\varepsilon-1) /[4(\varepsilon+1)]$, and $\varepsilon$ is the dielectric constant of liquid helium.

Equations (2) and (4) allow one to express the CR linewidth function $\gamma_{C R}(\omega)=2 v_{\text {eff }}(\omega)$ in terms of the equilibrium electron DSF

$$
\begin{gathered}
\gamma_{C R}(\omega)=\frac{[1-\exp (-\hbar \omega / T)]}{2 \hbar \omega m S_{A}} \times \\
\times \sum_{\mathbf{q}} q^{2} V_{q}^{2} \frac{\hbar q}{2 \rho \omega_{q}}\left[\left(N_{q}+1\right) S\left(q, \omega-\omega_{q}\right)+\right. \\
\left.+N_{q} S\left(q, \omega+\omega_{q}\right)\right] .
\end{gathered}
$$

Here $N_{q}$ is the ripplon distribution function. We use the conventional definition of the electron DSF:

$$
S(q, \omega)=N_{e}^{-1} \int \exp (i \omega t)\left\langle n_{\mathbf{q}}(t) n_{-\mathbf{q}}\right\rangle d t
$$

where $N_{e}$ is the number of electrons. Equation (7) can be applied to either state of the electron system: liquid or solid. In most cases, the resonance frequency $\omega$ is much higher than the typical ripplon frequency $\omega_{q}$, and the latter can be disregarded in the argument of the electron DSF.

\section{Wigner solid dynamical structure factor}

According to the previous Section, the determination of the CR line shape of a $2 \mathrm{D}$ electron system is reduced to evaluation of its equilibrium DSF. The important thing is that the Wigner solid consists of charged particles, and its dynamics is crucially affected by a strong normal magnetic field $B$. The magnetic field mixes the longitudinal and transverse phonon modes. According to [16], the excitation spectrum of the WS $\Omega_{p, k}$ (here $p=+,-$ ) is determined by the equation

$$
\begin{gathered}
2 \Omega_{ \pm, k}^{2}=\omega_{l, k}^{2}+\omega_{t, k}^{2}+\omega_{c}^{2} \pm \\
\pm \sqrt{\left(\omega_{l, k}^{2}+\omega_{t, k}^{2}+\omega_{c}^{2}\right)^{2}-4 \omega_{l, k}^{2} \omega_{t, k}^{2}} .
\end{gathered}
$$

For high cyclotron frequencies we disregard the presence of surface dimples beneath each electron. In the limiting case $\omega_{c}>>\omega_{l, k}$, the spectrum of the low-frequency mode decreases steadily with $B$ as $\Omega_{-, k} \simeq \omega_{l, k} \omega_{t, k} / \omega_{c} \propto 1 / B$, while the spectrum of the high-frequency mode $\Omega_{+, k}$ approaches $\omega_{c} \propto B$.

Althongh the electron displacement operator is also affected by the magnetic field, its general form can still be written in a conventional way:

$\mathbf{u}_{\mathbf{n}}=\sum_{p, \mathbf{k}}\left[\frac{\hbar}{2 N_{e} m \Omega_{p, k}}\right]^{1 / 2}\left[\mathbf{E}_{p, \mathbf{k}} a_{p, \mathbf{k}} \exp \left(i \mathbf{k} \mathbf{r}_{\mathbf{n}}\right)+\right.$ h.c. $]$.

In the presence of the magnetic field, the polarization vectors of the WS phonons $\mathbf{E}_{p, \mathbf{k}}$ are no longer orthonormalized [17]:

$$
\begin{aligned}
& \mathbf{E}_{-, \mathbf{k}}=\sin (\lambda) \mathbf{e}_{l, \mathbf{k}}+i \cos (\lambda) \frac{\Omega_{-, k}}{\omega_{t, k}} \mathbf{e}_{t, \mathbf{k}}, \\
& \mathbf{E}_{+, \mathbf{k}}=\cos (\lambda) \mathbf{e}_{l, \mathbf{k}}-i \sin (\lambda) \frac{\Omega_{+, k}}{\omega_{t, k}} \mathbf{e}_{t, \mathbf{k}},
\end{aligned}
$$

where $\mathbf{e}_{l, \mathbf{k}}$ and $\mathbf{e}_{t, \mathbf{k}}$ are the usual polarization vectors in the absence of the magnetic field, and

$$
\sin ^{2}(\lambda)=\frac{\omega_{t, k}^{2}-\Omega_{-, k}^{2}}{\Omega_{+, k}^{2}-\Omega_{-, k}^{2}} .
$$

It is easy to prove that $\left|\mathbf{E}_{+, \mathbf{k}}\right|^{2}+\left|\mathbf{E}_{-, \mathbf{k}}\right|^{2}=2$. In the strong magnetic field limit, $\left|\mathbf{E}_{-, \mathbf{k}}\right|^{2} \simeq\left(\omega_{l, k}^{2}+\right.$ $\left.+\omega_{t, k}^{2}\right) / \omega_{c}^{2} \rightarrow 0$, while $\left|\mathbf{E}_{+, \mathbf{k}}\right|^{2} \simeq 2$.

Following the procedure developed for the neutron scattering theory [18], the Wigner solid DSF can be written as

$$
S(q, \omega)=\sum_{\mathbf{n}} \exp \left(-i \mathbf{q} \mathbf{r}_{\mathbf{n}}\right) \times
$$

$$
\times \int_{-\infty}^{\infty} \exp \left\{i \omega t+2 q^{2} \sum_{p=+,-}\left[W_{p}(\mathbf{n}, t)-W_{p}(0,0)\right]\right\} d t
$$

where

$$
\begin{gathered}
W_{p}(\mathbf{n}, t)=\frac{\hbar}{8 N_{e} m} \sum_{\mathbf{k}} \frac{\left|\mathbf{E}_{p, \mathbf{k}}\right|^{2}}{\Omega_{p, k}} \times \\
\times\left\{\left(n_{p, k}+1\right) \mathrm{e}^{i\left(\mathbf{k r}_{\mathbf{n}}-\Omega_{p, k} t\right)}+n_{p, k} \mathrm{e}^{-i\left(\mathbf{k r}_{\mathbf{n}}-\Omega_{p, k} t\right)}\right\},
\end{gathered}
$$


$n_{p, k}$ is the WS phonon distribution function. Usually, the expansion of $W_{p}(\mathbf{n}, t)$ in $\Omega_{p, k} t$ is justified only for high temperature range $T>\theta_{D}$, where $\theta_{D}$ is the WS Debye temperature. In this case, the Debye-Waller function $2 q^{2} W_{p}(0,0)>>1$ and the main contribution into the integral of Eq. (12) comes from the region near $t=0$.

Another approximation is usually used for low temperatures, when $2 q^{2} W(\mathbf{n}, t)$ is small. In this case, the exponential of Eq. (12) is expanded in $2 q^{2} W(\mathbf{n}, t)$, giving an elastic term, one-phonon terms, and multi-phonon terms.

In Ref. 7 it was reasonably noted that for strong magnetic fields the frequency $\Omega_{-, k}$ is low, and one can expand the displacement correlators in $\Omega_{-, k} t$ even for temperatures $T \ll \theta_{D}$. Additionally, it was shown that one can use the incoherent approximation. Taking this approach, we find

$$
\begin{gathered}
2 q^{2}\left[W_{-}(0, t)-W_{-}(0,0)\right] \simeq-x_{q} \frac{\Gamma_{C}^{2}}{4 \hbar^{2}}\left(t^{2}+\frac{i t \hbar}{T}\right), \\
\Gamma_{C}^{2}=\frac{\hbar \omega_{c}}{N_{e}} \sum_{\mathbf{k}}\left|\mathbf{E}_{-, \mathbf{k}}\right|^{2} \hbar \Omega_{-, k}\left(2 n_{-, k}+1\right),
\end{gathered}
$$

where $x_{q}=q^{2} l^{2} / 2$ is the dimensionless parameter. At $\omega_{c}>>\omega_{l, k}$, we have $\Gamma_{C}=\sqrt{2} e E_{f}^{(0)} l$, with

$$
e E_{f}^{(0)}=\sqrt{\left(m T / N_{e}\right) \sum_{\mathbf{k}}\left(\omega_{l, k}^{2}+\omega_{t, k}^{2}\right)} .
$$

The quantity $E_{f}^{(0)}$ is a measure of the average fluctuational electric field acting on an electron. The most important physics comes from the first term of Eq. (14), proportional to $t^{2}$. It is due to this term, affecting the time integral of Eq. (12), the conventional energy-conservation $\delta$ function is replaced by a heavily broadened Gaussian function.

For the high-frequency mode one can assume $n_{+, k}<<1$ and $\left|\mathbf{E}_{+, \mathbf{k}}\right|^{2} \simeq 2$, which gives

$$
2 q^{2} W_{+}(0, t) \simeq \frac{x_{q}}{N_{e}} \sum_{k} \frac{\omega_{c}}{\Omega_{+, k}} \exp \left(-i \Omega_{+, k} t\right),
$$

and $2 q^{2} W_{+}(0,0) \simeq x_{q}$, where $\Omega_{+, k} \simeq \omega_{c}$. Therefore, we can keep the high-frequency Debye-Waller factor exp $\left(-x_{q}\right)$ as it is and expand the DSF in $2 q^{2} W_{+}(0, t)$, a procedure which is similar to the conventional low-temperature expansion. Thus, we have multi-phonon emission terms for the high-frequency mode. Usually, the scattering events described by terms proportional to $\exp \left(-i N \omega_{c} t\right)$ are forbidden at low temperatures $T \ll \hbar \omega_{c}$ (except for the term with $N=1$ ), since a ripplon or a vapor atom does not have enough energy in the laboratory frame for such emission. In our case, the multi-phonon emission of high-frequency phonons is possible because of the strong broadening introduced by the low-frequency mode [Eq. (14)]. Such emission is accompanied by the creation and annihilation of a great number of low-frequency phonons.

A straightforward evaluation of all terms of the expansion in $2 q^{2} W_{+}(0, t)$ gives the following result:

$$
\begin{gathered}
S(q, \omega)=\frac{2 \sqrt{\pi} \hbar}{\Gamma_{C}} \times \\
\times \sum_{N=0}^{\infty} \frac{x_{q}^{N-1 / 2}}{N !} \exp \left[-x_{q}-\frac{\hbar^{2}\left(\omega-N \omega_{c}\right)^{2}}{x_{q} \Gamma_{C}^{2}}\right] .
\end{gathered}
$$

We have disregarded the small frequency shift caused by the second term of Eq. (14). Contrary to Ref. 7, here the WS DSF involves many quanta of cyclotron motion $\left(N \omega_{c}\right)$. Surprisingly, Eq. (17) evaluated for the electron solid state coincides with the DSF of a highly correlated electron liquid [11] in which the electron motion is affected by the quasi-uniform fluctuational electric field $E_{f}$ distributed as a Gaussian with $\sqrt{\left\langle E_{f}^{2}\right\rangle}=E_{f}^{(0)}$ and the collision broadening of Landau levels $\Gamma_{N}$ is disregarded $\left(\Gamma_{C} \gg \Gamma_{N}\right)$. The quantity $\Gamma_{C}$ can be called the Coulomb broadening of the electron DSF, but it has no relation with the Landau level broadening. Moreover, according to [10,11], the quasi-uniform fluctuational electric field reduces the collision broadening of Landau levels. The result of Eq. (17) explains the experimental observation reported in Ref. 9 that the WS transition does not affect the CR linewidth.

The first term $(N=0)$ of Eq. (17) describes the DC magnetoconductivity of the WS. In the extreme limit $\Gamma_{C}>>\Gamma_{N}$, the electron DSF $S(q, 0) \propto$ $\propto 1 / \Gamma_{C} \propto n_{s}^{-3 / 4}$, which decreases the effective collision frequency and $\sigma_{x x}$.

When the fluctuational field is weak $\left(\Gamma_{C}<<\hbar \omega_{c}\right)$, the $\mathrm{CR}$ linewidth is determined by the term with $N=1$. The frequency dependence of $\gamma_{C R}(\omega)$ affects the CR line shape. As a result, the CR absorption approaches the Lorentzian shape only in the limit $\Gamma_{C}>>\gamma_{C R}\left(\omega_{C}\right)$ where $\gamma_{C R}(\omega) \simeq$ const. In spite of the frequency dependence of the parameter $\gamma_{C R}$, the linewidth of the absorption curve at the half-height is very close to $\gamma_{C R}\left(\omega_{c}\right)$. Increasing the fluctuational field $E_{f}^{(0)}$ makes nonlinear terms $(N \neq 1)$ important, changing significantly the density dependence of the linewidth. 


\section{Results and discussions}

Employing the Wigner solid DSF found above, the CR linewidth $\gamma_{C R}\left(\omega_{c}\right)$ can be written as

$$
\begin{gathered}
\gamma_{C R}\left(\omega_{C}\right)=\frac{\left(e E_{\perp}\right)^{2}[1-\exp (-\hbar \omega / T)] T}{2 \sqrt{\pi} \hbar \alpha \Gamma_{C}} \times \\
\times \sum_{N=0}^{\infty} \frac{1}{N !} F_{N}\left(\hbar \omega_{C}|N-1| / \Gamma_{C}\right),
\end{gathered}
$$

where

$$
\begin{gathered}
F_{N}(\eta)=\int_{0}^{\infty} x^{N-1 / 2} V_{S}^{2}(x) \exp \left(-x-\eta^{2} / x\right) d x \\
V_{S}(x)=1+\frac{\Lambda x}{e E_{\perp} l^{2}} w\left(\frac{x}{2 \gamma^{2} l^{2}}\right),
\end{gathered}
$$

the coupling function $w(x)$ is defined in Eq. (6). For small broadening of the WS DSF $\left(\Gamma_{C}<<\hbar \omega_{c}\right)$, the main contribution into Eq. (18) is given by the term with $N=1$. Since $F_{1}(0)$ does not depend directly on the electron density $n_{s}$, the CR linewidth determined by this term decreases with electron density $\gamma_{C R}\left(\omega_{C}\right) \propto 1 / \Gamma_{C}$, in accordance with Ref. 7.

For strong Coulomb broadening of the DSF $\left(\Gamma_{C} \sim \hbar \omega_{c}\right)$ terms with $N \neq 1$ play an important role, changing the sign of the many-electron effect on the CR linewidth: a decrease of the CR linewidth is turned into an increase. Therefore, the same Coulombic effect (broadening of the electron DSF) leads to the successive narrowing and broadening of the CR linewidth.

Under conditions of the experiment of Ref. 9, the Debye frequency of WS phonons $\omega_{D} \geq \omega_{c}$ and therefore the requirement $\omega_{l, k}<\omega_{c}$ is met only for the long-wavelength part of the phonon spectrum. According to Eq. (14), in this regime $\Gamma_{C} \gtrsim \hbar \omega_{c}$ and the terms of Eq. (18) with $N \gg 1$ should be dominant. In evaluating Eq. (18) numerically we use $E_{f}^{(0)}$ found by Monte Carlo simulations [19] for a broad range of the Coulomb coupling parameter $e^{2} \sqrt{\pi n_{s}} / T$. The full linewidth of the CR calculated according to Eq. (18) is shown in Fig. 1. When varying the electron density $n_{s}$ the holding field is fixed at $E_{\perp}=275 \mathrm{~V} / \mathrm{cm}$. It is clear that the contribution from the linear term (the approximation of Ref. 7 - dashed curve) cannot explain the experimental data. On the other hand, the contribution from all terms (solid curve) behaves in good accord-

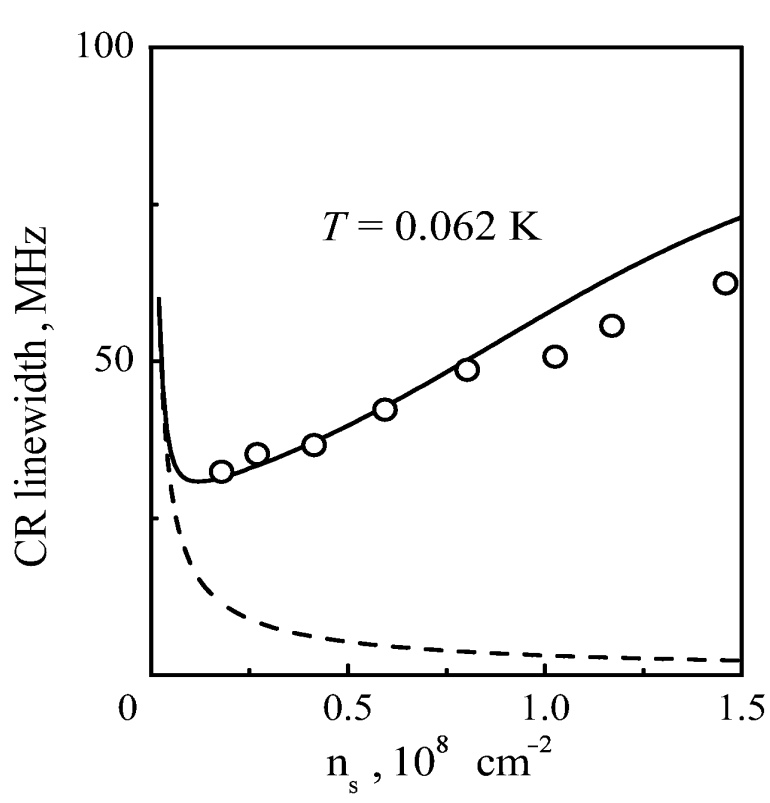

Fig. 1. CR linewidth vs $n_{s}$ : data from Ref. 9 (circles), WS theory [Eq. (18)] (solid curve), approximation of Ref. 7 (dashed curve)

ance with the data. The deviations of the experimental data from the proposed model appearing at high densities are assumed to be caused by the substantial increase of the WS Debye frequency $\omega_{D}>\omega_{c}$ and $\Omega_{+, k}$ for the short-wavelength part of the phonon spectrum.

It is interesting to compare the new WS theory with the old linewidth vs $E_{\perp}$ data of Refs. 5, 6, where the electron density $n_{s}$ was varied according to the saturation condition $E_{\perp}=2 \pi e n_{s}$. For comparably high temperatures $(T=0.72 \mathrm{~K})$, in addition to the electron-ripplon scattering, we take into account the electron interaction with vapor atoms. The half-width data and the corresponding theoretical curves are shown in Fig. 2. The theoretical curve, including all nonlinear terms $\exp \left(i N \omega_{c} t\right)$ (solid curve), describes the data nicely. For the low temperature regime of Fig. $3(T=0.4 \mathrm{~K})$ the interaction with vapor atoms can be disregarded. Both figures (2 and 3) show that the contribution from terms with $N>1$ is crucial for strong holding fields (high electron densities). The holding field dependence of the electron-ripplon coupling $V_{q}$ is not strong enough to explain the sharp increase of the linewidth data, taking into account the only linear term with $N=1$ (dash curves). The inclusion of many cyclotron motion quanta $N \omega_{c}(N>>1)$ makes the WS theory (solid curves) close to the data and the half-width behavior similar to the one of the semi-classical half-width (dotted curves).

It is instructive to note that exciting many highfrequency phonons with energy $\sum \hbar \Omega_{+, k}>N \hbar \omega_{c}$ in 


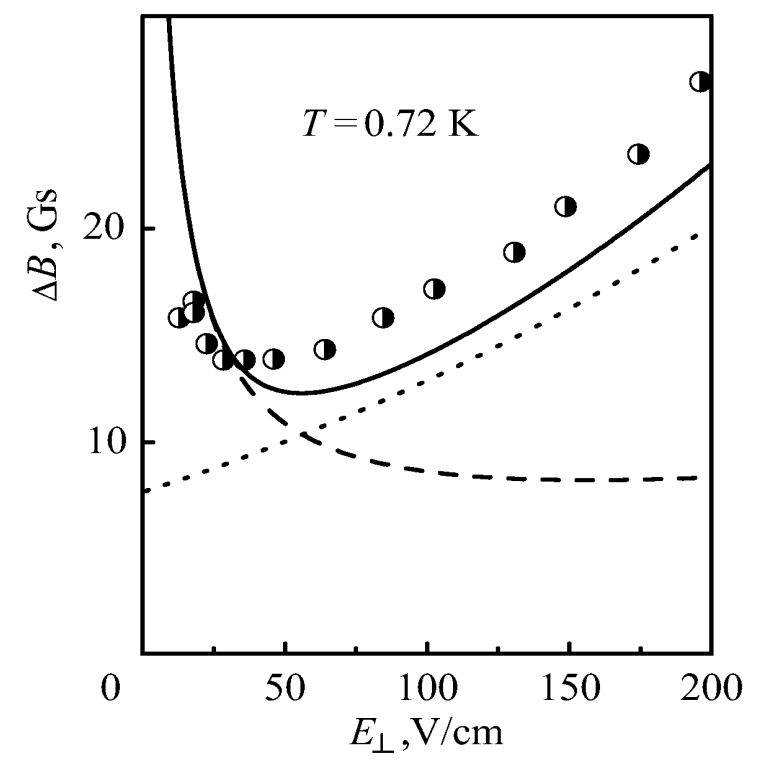

Fig. 2. CR half-width $\Delta B$ vs the holding electric field $\left(E_{\perp}=2 \pi e n_{s}\right)$ : data from Ref. 5 (circles), WS theory [Eq. (18)] (solid curve), approximation of Ref. 7 (dashed curve), semiclassical theory (dotted curve)

a single scattering event involving a ripplon or a vapor atom is a pure many-electron effect, since at low temperatures $T<\hbar \omega_{c}$ neither a scatterer nor an electron have enough energy for this excitation. In a liquid state of the electron system, it is a quasiuniform electric field $E_{f}$ created by many other electrons that makes the electron scattering inelastic in the frame moving along with an electron orbit center. In this frame, a heavy helium vapor atom moving with the velocity $-\mathbf{u}_{f}$ (here $\mathbf{u}_{f}$ is the drift velocity of an electron orbit induced by $E_{f}$ ) have enough kinetic energy, and electron scattering on higher Landau levels becomes possible if the energy exchange in the moving frame $\hbar \mathbf{q u} \mathbf{u}_{f} \sim e E_{f} l$ is larger than the level spacing $\hbar \omega_{c}$.

In the WS theory, it is the inclusion of a great number of low-frequency WS phonons $\left(\Omega_{-, k}<T / \hbar\right)$ with the distribution function $n_{-, k} \simeq T / \hbar \Omega_{-, k}>>1$ that makes possible the emission of many high frequency phonons. One can find the proper multiphonon terms with conventional $\delta$ functions by expanding the exponential of Eq. (12) in both $2 q^{2} W_{-}(\mathbf{n}, t)$ and $2 q^{2} W_{+}(\mathbf{n}, t)$. The explanations given just above describe a complicated phenomenon, involving not only many electrons but also a great number of WS excitations, in terms of simple scattering events. Still, the quantity in Eq. (17) represents the DSF of the electron crystal which does not depend on the interaction with scatterers.

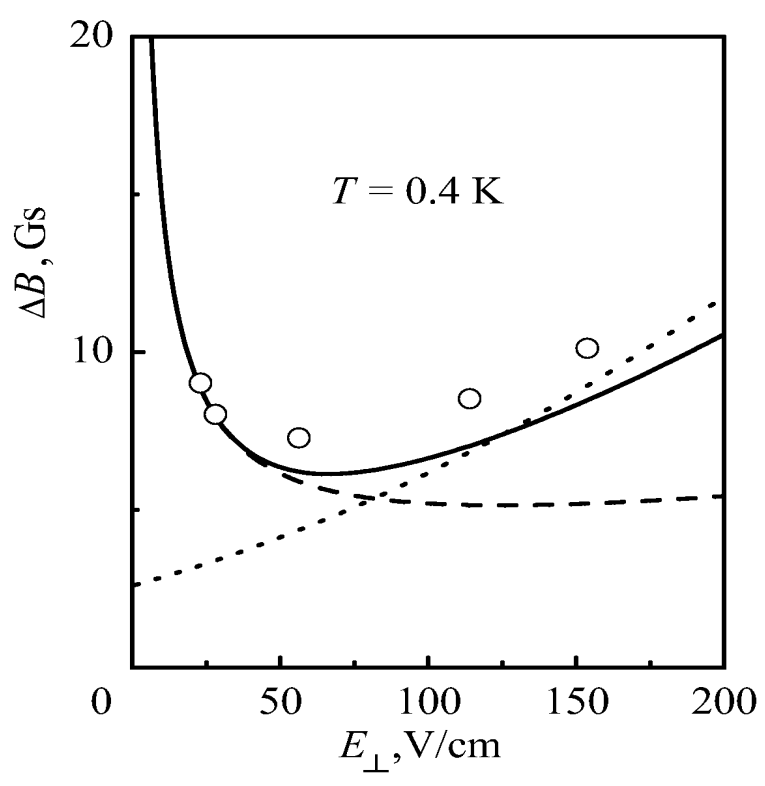

Fig. 3. Low temperature CR half-width data and theory. The notation is the same as in Fig. 2.

\section{Conclusions}

Establishing a relation between the effective collision frequency and the electron dynamical structure factor, the conductivity theory of the Wigner solid presented here takes advantage of methods developed for the theory of thermal neutron scattering. The important point is that a $2 \mathrm{D}$ electron system represents a moving target whose DSF is analyzed, while scatterers (ripplons and vapor atoms) play the role of thermal particle fluxes, since their properties are well known.

The existence of strongly separated phonon modes of the electron crystal in a high magnetic field $\left[\Omega_{-, k}<T / \hbar\right.$ and $\left.\Omega_{+, k}>\omega_{c}>>T / \hbar\right]$ allows one to combine the conventional high-temperature approximation for the solid DSF (applied to the low-frequency mode only) and an exact evaluation of the contribution from the high-frequency mode. The low-frequency mode is shown to affect crucially the result that comes out for the high-frequency mode, causing a strong broadening of the DSF and strengthening the contribution from multi-phonon terms.

We have shown that under the conditions of the CR experiments with SEs conducted up till the present [5,6,9] the many-electron effect is very strong, and the multi-phonon emission terms of the high-frequency mode disregarded previously can dominate the usual one-phonon term. The inclusion of all these terms leads to a DSF of the Wigner solid which practically coincides with the DSF of a $2 \mathrm{D}$ electron liquid whose properties are affected by a quasi-uniform fluctuational electric field $E_{f}$ of the 
proper strength [11]. The CR linewidth calculated for the electron-ripplon scattering regime agrees well with the data of Refs. 5, 6, 9, eliminating the discrepancy between theory and experiment reported in Ref. 9.

A part of this work was supported by the INTAS97-1643 grant.

1. C. C. Grimes and G. Adams, Phys. Rev. Lett. 42, 795 (1979).

2. D. S. Fisher, B. I. Halperin, and P. M. Platzman, Phys. Rev. Lett. 42, 798 (1979).

3. B. A. Wilson, S. J. Allen, Jr., and D. C. Tsui, Phys. Rev. Lett. 44, 479 (1980); B.A. Wilson, S. J. Allen, Jr., and D. C. Tsui, Phys. Rev. B24, 5887 (1981).

4. Z. Schlesinger, W. I. Wang, and A. H. MacDonald, Phys. Rev. Lett. 58, 73 (1987).

5. V. S. Edel'man, Zh. Eksp. Teor. Fiz. 77, 673 (1979) [Sov. Phys. JETP 50, 338 (1979)].

6. V. S. Edel'man, Usp. Fiz. Nauk 130, 675 (1980) [Sov. Phys. Usp. 23, 227 (1980)].

7. M. I. Dykman, J. Phys. C15, 7397 (1982).
8. M. Saitoh, J. Phys. Soc. Jpn. 56, 706 (1987).

9. L. Wilen and R. Giannetta, Phys. Rev. Lett. 60, 231 (1988).

10. E. Teske, Yu. P. Monarkha, M. Seck, and P. Wyder, Phys. Rev. Lett. 82, 2772 (1999).

11. Yu. P. Monarkha, E. Teske, and P. Wyder, Phys. Rev. B62, 2593 (2000).

12. W. Gotze and J. Hajdu, J. Phys. C11, 3993 (1978).

13. Y. Shiwa and A. Isihara, J. Phys. C16, 4853 (1983).

14. Yu. P. Monarkha and K. Kono, submitted for publication.

15. Yu. P. Monarkha, in: Two-Dimensional Electron Systems on Helium and Other Cryogenic Substrates, E. Y. Andrei (ed.), Kluver Academic Publishers, Dordrecht, Boston, London (1997).

16. A. V. Chaplik, Zh. Eksp. Teor. Fiz. 62, 746 (1972) [Sov. Phys. JETP 35, 395 (1972)].

17. Yu. P. Monarkha and S. S. Sokolov, Fiz. Nizk. Temp. 8, 350 (1982) [Sov. J. Low Temp. Phys. 8, 173 (1982)].

18. W. Marshall and S. W. Lovesey, Theory of Thermal Neutron Scattering, Oxford, Clarendon Press (1971).

19. C. Fang-Yen, M. I. Dykman, and M. J. Lea, Phys. Rev. B55, 16272 (1997). 